\title{
Second Primary Malignancy Risk After Radioactive lodine Treatment for Thyroid Cancer: A Systematic Review and Meta-analysis
}

\author{
Anna M. Sawka, M.D., Ph.D., FRCPC, ${ }^{1,2}$ Lehana Thabane, Ph.D., ${ }^{3,4}$ Luciana Parlea, M.D., ${ }^{2}$ \\ Irada Ibrahim-Zada, M.D., ${ }^{5}$ Richard W. Tsang, M.D., FRCPC, ${ }^{6}$ James D. Brierley, M.D., FRCPC, ${ }^{6}$ \\ Sharon Straus, M.D., FRCPC, M.Sc., ${ }^{7}$ Shereen Ezzat, M.D., ${ }^{8-10}$ and David P. Goldstein, M.D., FRCSC ${ }^{11,12}$
}

Background: The risk of second primary malignancies (SPMs) associated with cancer therapies is an important concern of thyroid cancer survivors and physicians. Our objective was to determine if the risk of SPMs is increased in individuals with thyroid cancer treated with radioactive iodine (RAI), compared to those not treated with RAI. Methods: We performed a systematic review of the literature and meta-analysis. Two independent reviewers screened citations and reviewed full-text papers. If not reported by the primary authors, the relative risk (RR) of SPMs was calculated by dividing the standardized incidence ratio of SPM in individuals with thyroid cancer treated with RAI compared to those not treated with RAI (with associated 95\% confidence intervals [CI]). The natural logarithms of RRs of respective SPMs, weighted by the inverse of the variance, were pooled using fixed effects models and the exponential of the results was reported.

Results: Two multi-center studies (one from Europe and the other from North America) were included in this review. The RR of SPMs in thyroid cancer survivors treated with RAI was significantly increased at $1.19(95 \%$ confidence interval $[\mathrm{CI}] 1.04,1.36, p=0.010$ ), relative to thyroid cancer survivors not treated with RAI (data from 16,502 individuals), using a minimum latency period of 2 to 3 years after thyroid cancer diagnosis. The RR of leukemia was also significantly increased in thyroid cancer survivors treated with RAI, with an RR of 2.5 (95\% CI $1.13,5.53, p=0.024)$. We did not observe a significantly increased risk of the following cancers related to prior RAI treatment: bladder, breast, central nervous system, colon and rectum, digestive tract, stomach, pancreas, kidney (and renal pelvis), lung, or melanoma of skin.

Conclusions: The risk of SPMs in thyroid cancer survivors treated with RAI is slightly increased compared to thyroid cancer survivors not treated with RAI.

\section{Introduction}

$\mathrm{T}$

HYROID CANCER is the most commonly diagnosed endocrine malignancy $(1,2)$. Based on statistics reported in 2008, approximately 4300 Canadians (1) and 37,340
Americans (2) were diagnosed with thyroid cancer in the prior year. Furthermore, the incidence in thyroid cancer is rising throughout the world (1-5). In Canada, the incidence of thyroid cancer has risen by $5.5 \%$ per year in men and by $10.1 \%$ per year in women since 1997 (1). Similar trends in increasing

\footnotetext{
${ }^{1}$ Division of Endocrinology and Department of Medicine, University Health Network, Toronto, Ontario, Canada.

${ }^{2}$ Division of Endocrinology and Department of Medicine, University of Toronto, Toronto, Ontario, Canada.

${ }^{3}$ Department of Clinical Epidemiology and Biostatistics, McMaster University, Hamilton, Ontario, Canada.

${ }^{4}$ Centre for Evaluation of Medicines, St. Joseph's Healthcare, Hamilton, Ontario, Canada.

${ }^{5}$ Division of Endocrinology, Department of Medicine, University Health Network, Toronto, Ontario, Canada.

${ }^{6}$ Department of Radiation Oncology, University Health Network and University of Toronto, Toronto, Ontario, Canada.

${ }^{7}$ Department of Knowledge Translation, University of Toronto, Ontario, Canada.

${ }^{8}$ Division of Endocrinology and Department of Medicine, Mount Sinai Hospital, Toronto, Ontario, Canada.

${ }^{9}$ The Freeman Centre for Endocrine Oncology, Toronto, Ontario, Canada.

${ }^{10}$ The Ontario Cancer Institute, Toronto, Ontario, Canada.

${ }^{11}$ Department of Surgery, University Health Network and University of Toronto, Toronto, Ontario, Canada.

${ }^{12}$ Department of Otolaryngology Head and Neck Surgery, University Health Network and University of Toronto, Toronto, Ontario, Canada.
} 
numbers of individuals being diagnosed with thyroid cancer have been reported in the United States (2-4) and the United Kingdom (5). Yet the relative 5-year survival rate for thyroid cancer is among the highest of all cancers in Canada (98\%) (1) and the United States (96.7\%) (2). Recent major and sustained improvements in thyroid cancer-related survival have also been reported in Europe from 1991 to 2002 (6). The most common histologic subtype of thyroid cancer is papillary carcinoma, accounting for about $80 \%$ of cases, followed by follicular carcinoma (collectively referred to as welldifferentiated thyroid carcinoma) $(7,8)$.

Primary treatment of well-differentiated thyroid carcinoma includes thyroidectomy, thyroid hormone treatment, and selectively, post-surgical radioactive iodine (RAI) depending on patient and disease features such as pathologic stage (9-15). The use of RAI in the treatment of thyroid carcinoma is relatively common, with the National Thyroid Cancer Treatment Co-operative Group recently reporting that RAI treatment had been administered in $62-75 \%$ of individuals in their studied cohorts (16).

We recently reported that thyroid cancer survivors are at increased risk of developing second primary malignancies (SPMs) relative to the general population (17). In the current study, we have performed a systematic review and metaanalysis examining whether individuals with thyroid cancer treated with RAI are at higher risk of developing SPMs compared to individuals with thyroid cancer not treated with RAI.

\section{Patients and Methods}

\section{Inclusion and exclusion criteria for studies}

We restricted this review to published English-language studies comparing the risk of SPM in individuals with thyroid cancer treated with RAI compared to individuals with thyroid cancer not treated with RAI. Second primary malignancies were required to have been diagnosed after an initial diagnosis of thyroid cancer and recurrences of thyroid cancer were not considered SPMs. A requirement for inclusion of the study was the presentation of a relative risk (RR), odds ratio, or hazard ratio (with respective $95 \%$ confidence intervals, CIs) comparing SPM risk in thyroid cancer patients treated with RAI to those not treated with RAI, or reporting of sufficient information such that ratios and accompanying 95\% CIs could be calculated. Published studies from cohort studies, crosssectional studies, cancer registries, administrative datasets, meta-analyses, and large case series (including $>50$ patients) were eligible for inclusion. A minimum of 50 thyroid carcinoma patients was required be included in a study, for meaningful analysis of SPMs. In the case of multiple studies on the same population or overlapping populations, the largest or the most completely reported of the duplicate studies examining the intervention and outcome of interest was included.

\section{Description of the search for relevant studies}

An electronic search was conducted for citations on SPMs in thyroid cancer survivors using the following electronic databases (no language restriction) (updated December 2006; March 31, 2007; and September 20, 2008): Ovid Medline (beginning 1966), Ovid Medline-in-Process and non-indexed citations, Cochrane database of systematic reviews, American
College of Physicians Journal Club, All Evidence-Based Medicine Reviews (including Database of Abstracts of Reviews of Effects and Cochrane Central Register of Controlled Trials), EMBASE (beginning 1980), Cumulative Index to Nursing and Allied Health Literature (beginning 1982), and Ovid Healthstar (beginning 1966). We also cross-referenced potentially relevant reviewed studies and contacted experts for relevant citations. Otherwise, non-English citations were excluded from this review because of the lack of resources for translation, although if an English abstract was available, this was used to judge potential relevance.

\section{Selection of studies and data abstraction}

All abstracts and citations were reviewed independently by two reviewers. Any abstract or citation deemed potentially relevant by either reviewer was retrieved and reviewed in full-text form by two independent reviewers. Any potentially relevant full-text papers retrieved from other sources, such as expert suggestion or cross-referencing of reviewed papers, were also subjected to review by two independent reviewers. We achieved consensus between reviewers for all studies included in the final systematic review. Data were independently abstracted by two abstractors, and the final data was reviewed by a third party for accuracy. Any discrepancies in data abstraction were resolved by consensus among the third party and the data abstractors.

\section{Statistical analyses}

Kappa statistics were calculated to assess agreement between reviewers for relevance at the abstract review stage and for inclusion at the full-text review stage, prior to achieving consensus. The kappa statistics and 95\% CIs were calculated using CIA software (2nd edition, BMJ Books, London, UK). The primary analysis was a pooled analysis examining the RR for any SPM in thyroid cancer survivors treated with RAI compared to thyroid cancer survivors not treated with RAI. Secondary pooled analyses of RRs for respective specific SPMs were also performed. If not reported by the primary authors, the RR of SPMs was calculated by dividing the standardized incidence ratio (observed divided by expected cases) of SPM in individuals with thyroid cancer treated with RAI compared to those not treated with RAI (with associated 95\% CIs) (CIA software). Estimates of the natural logarithm of the RRs weighted by the inverse of the variance were pooled using fixed effects models and the results were exponentiated to convert them into the RR scale (Comprehensive MetaAnalysis software, version 2.0; Biostat, Englewood, NJ). Both the pooled estimate of RR and corresponding 95\% CI are reported. The robustness of these analyses was checked using random effects models. A minimum of 1-year latency period from time of diagnosis of thyroid cancer or RAI treatment to diagnosis of SPM was selected for pooled analyses, with effort to pool data with similar latency periods. Thus for included studies, regardless of total follow-up period, only nonthyroidal malignancies diagnosed 1 year or later after the primary diagnosis of thyroid cancer were counted as SPMs in the pooled analyses. If multiple analyses using various latency periods were reported in primary studies, we only pooled data utilizing latency periods 1 year or longer after the initial diagnosis of thyroid cancer (the shortest latency period, 1 year or longer after diagnosis of thyroid cancer). The rationale for 
selecting a latency period of at least 1 year was based on the theoretical concept that it takes some time for biologic processes to result in development and detection of a cancer after exposure to a potential carcinogen. Heterogeneity of data included in the pooled primary analysis was estimated by calculation of an I-squared value (variation in effect size attributed to heterogeneity) and a tau-squared value for between-study variance (Comprehensive Meta Analysis software, version 2.0).

\section{Results}

\section{Description of the studies}

We retrieved 1571 potentially relevant citations using our electronic search and an additional 36 citations from other sources, for a total of 1607 reviewed publications (Fig. 1). Agreement between reviewers for relevance of 1571 abstracts and citations retrieved by the electronic search was reflected by a kappa statistic of 0.56 (95\% CI 0.46, 0.65). The kappa statistic was $0.88(95 \%$ CI $0.79,0.97)$ for the initial review of 172 full-text papers. After considering the inclusion and exclusion criteria and excluding studies of duplicate populations (which were smaller, less recent, or contained insufficient information for calculation of RR related to RAI treatment in thyroid cancer), only two studies were included in the review $(18,19)$ (Fig. 1).

The characteristics of the included studies by Brown et al. (18) and Rubino et al. (19) are described in Table 1. The population in the study by Brown et al. (18) was assembled using cancer registry records from the Surveillance, Epidemiology, and End Results (SEER) program of the National Cancer Institute in North America. In contrast, the population in the study by Rubino et al. (19) was assembled using data from three European clinical cohorts (Sweden 1951-1997, Italy 1958-1995, and France 1934-1995). In both papers, the study population was comprised of survivors of papillary or follicular thyroid carcinoma, and individuals who had been diagnosed with another primary malignancy prior to the thyroid were excluded $(18,19)$. The median or mean age of diagnosis of thyroid cancer was in the early 40 s for both studies $(18,19)$. The overall median follow-up periods were as follows: 8.6 years in the SEER study (18) and 13 years in the European study (19). The stage of thyroid cancer at diagnosis was not reported in either study $(18,19)$. In the SEER study, overall $33.9 \%$ of individuals received radioisotope therapy, whereas $6.6 \%$ received either a combination of radioisotope therapy with external beam radiotherapy, external beam radiotherapy alone, or brachytherapy (18). In the European study, $62 \%$ of individuals received RAI, $17 \%$ received external beam radiotherapy, and $9 \%$ received both (19). The mean cumulative activity of RAI received was not reported in SEER (18) but was 6.0 GBq (range, 0.2-55.5 GBq) in the European study. The overall percentage of individuals developing one or more SPMs in each of the studies was as follows: SEER $7.1 \%$ $(2158 / 30,278)(18)$ and European cohorts 8.4\% (576/6841) (19). The median time to development of an SPM after a thyroid cancer diagnosis was 8.1 years (range, 0.2-29.7 years) in the SEER study (18). In the European study, the mean time from thyroid cancer diagnosis to development of an SPM was 15 years (range, 2-55 years) (19).

To assess study quality, we examined all studies with respect to four variables, including reporting of mode of data collection (retrospective or prospective), numerical description of the eligible participants excluded from the study, numerical description of losses to follow-up in the study, and independent confirmation of thyroid cancer and SPM diagnoses. In both of the included studies, it was not explicitly stated that data were prospectively collected $(18,19)$; however, we assumed that the cancer registry data collected in the SEER database in the Brown et al. (18) study was likely

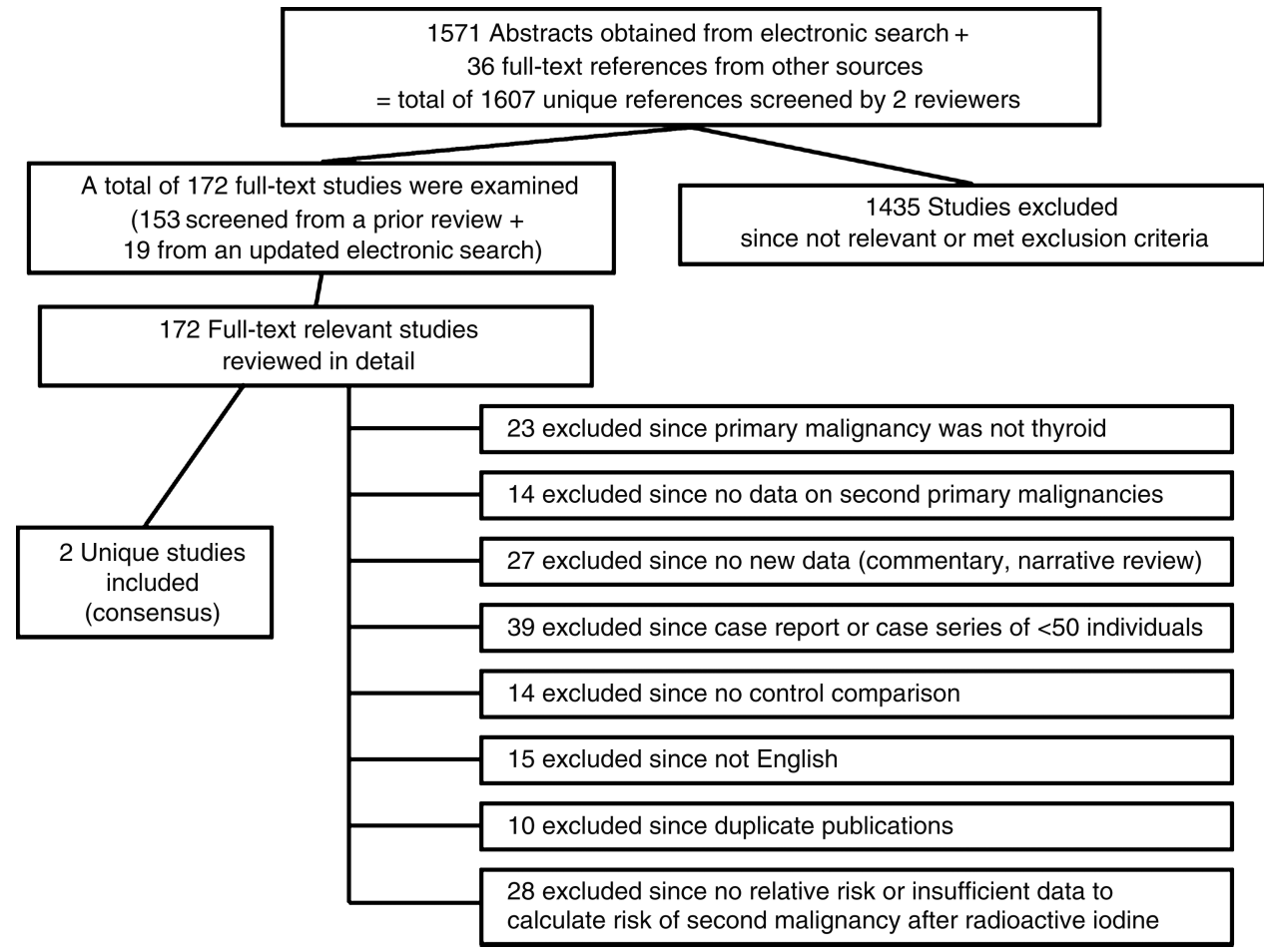

FIG. 1. Process of study selection for the systematic review. 
Table 1. Characteristics of the InCluded Studies

\begin{tabular}{|c|c|c|c|c|c|}
\hline Study & Type of dataset & $\begin{array}{c}\text { Time period } \\
\text { of study } \\
\text { (period for RAI RR }^{\text {SPM analysis) }}\end{array}$ & $\begin{array}{l}\text { Years of } \\
\text { follow-up } \\
\text { (range) }\end{array}$ & $\begin{array}{l}\text { Age at diagnosis of } \\
\text { thyroid cancer (range) }\end{array}$ & $\begin{array}{l}\text { No. of thyroid } \\
\text { cancer survivors } \\
\text { (no. in RAI RR } \\
\text { SPM analysis) }\end{array}$ \\
\hline $\begin{array}{l}\text { Brown } \\
\quad \text { et al., } 2008(18)\end{array}$ & $\begin{array}{l}\text { North American } \\
\text { registry cohorts }\end{array}$ & $\begin{array}{c}1973-2002 \\
(1988-2002)\end{array}$ & $\begin{array}{c}\text { Median } 8.6 \\
(0.2-29.7)\end{array}$ & $\begin{array}{l}\text { Median } 42 \\
\quad(4-100)\end{array}$ & $\begin{array}{c}30,278 \\
(9661 ; 4248 \text { received RAI })^{\mathrm{c}}\end{array}$ \\
\hline $\begin{array}{l}\text { Rubino } \\
\text { et al., } 2003 \text { (19) }\end{array}$ & $\begin{array}{l}\text { European clinical } \\
\text { cohorts }\end{array}$ & $\begin{array}{c}\text { France 1934-1995 } \\
\text { Sweden 1951-1977 } \\
\text { Italy 1958-1995 } \\
\text { (same as above) }\end{array}$ & $\begin{array}{l}\text { Mean } 13 \\
(2-55)\end{array}$ & $\begin{array}{l}\text { Mean } 44 \\
\quad(2-91)\end{array}$ & $\begin{array}{c}6,841 \\
(6841 ; 4225 \text { received RAI })^{d}\end{array}$ \\
\hline
\end{tabular}

${ }^{a}$ Details relating to the data used for the calculation of the pooled RR of SPMs after RAI treatment (compared to no RAI treatment).

${ }^{\mathrm{b}}$ In the case of the Brown et al. study (18), the data for the pooled analysis was restricted to a subgroup from 1988 to 2002 , when details of radioisotope use were reported, using a latency period cut-off of 3 years. Therapeutic radioiosotope use in thyroid cancer was assumed to be RAI treatment.

${ }^{\mathrm{c}}$ For the Brown et al. study (18), the latency period (the minimum time from thyroid cancer diagnosis) to development of a SPM was 3 years for the pooled analyses. Thus, only second cancers diagnosed a minimum of 3 years after the initial thyroid cancer diagnosis were counted as SPMs in the pooled analysis.

${ }^{\mathrm{d}}$ For the Rubino et al. study (19), the latency period (the minimum time from thyroid cancer diagnosis) to development of a SPM was 2 years for the pooled analyses. Thus only second cancers diagnosed a minimum of 2 years after the initial thyroid cancer diagnosis were counted as SPMs in the pooled analysis.

$\mathrm{RR}$, relative risk; SPMs, second primary malignancies; RAI, radioactive iodine.

prospectively collected. In the European study, the term cohort was used, implying a longitudinal design (19). In both included studies, there was no clear numerical description of the ineligible thyroid cancer survivors excluded from the study $(18,19)$. Losses to follow-up were not reported in the SEER study (18) but were reported to be approximately $20 \%$ in the European study (19). It was not clearly reported whether there was any independent confirmation of cancer diagnoses in the SEER study, although the authors indicated that SEER mandates a $98 \%$ case ascertainment and that quality and completeness studies are conducted yearly in SEER areas (18). In the European study, the authors reported that histologic diagnoses were obtained for all second primaries to exclude the possibility of metastases, although it was not clear if there were any independent histologic reviews (19).

\section{Results of the pooled analyses}

In our meta-analyses, we pooled data on the RR of SPMs diagnosed a minimum of 3 years after the initial diagnosis of thyroid cancer from the SEER database (18) with RR data for SPMs diagnosed a minimum of 2 years after thyroid cancer diagnosis in the European study (19) (as the populations and latency periods were judged to be sufficiently similar for analysis). In the SEER study, data from the years 1988 to 2002 were used for the pooled analysis, since in this period, radioisotope therapy was distinguished from other types of radiation treatment (18). We assumed that radioisotope therapy for differentiated thyroid cancer reported in the SEER study (18) was RAI $\left({ }^{131} \mathrm{I}\right)$. For the SEER data (18), we estimated the RR of SPMs in RAI-treated thyroid cancer survivors relative to thyroid cancer survivors not treated with RAI by dividing the observed or expected rate of SPMs in the RAI group by that in the non-RAI group. In our pooled analysis of data from SEER and the European study, the RR of SPMs in thyroid cancer survivors treated with RAI was significantly increased at 1.19 (95\% confidence interval [CI] 1.04, 1.36, $p=0.010)$, relative to thyroid cancer survivors not treated with RAI (data from 16,502 individuals) (Table 2, Fig. 2). There was significant heterogeneity of RRs of any SPM in the pooled analysis (I-squared $<0.001$, tau-squared $<0.001$ ). We found that the pooled RR of leukemia was increased in thyroid cancer survivors treated with RAI, with an RR of 2.50 $(95 \%$ CI 1.13, 5.53, $p=0.024)$ relative to those not treated with RAI. We did not observe a significantly increased pooled RR of the following cancers related to prior RAI treatment: bladder, breast, central nervous system, colon and rectum, digestive tract, stomach, pancreas, kidney (and renal pelvis), lung, and melanoma of skin. The significance level of the findings was robust for all analyses using respective random effects models.

It is important to note that in the European study, an increased risk of the following SPMs was reported in RAItreated thyroid cancer survivors relative to those not treated with RAI: salivary glands (RR 7.5, 95\% CI 1.2-143), bone and soft tissue (RR 4.0, 1.5-12.4), female genital organs (RR 2.2, 95\% CI 1.3-3.9), and uterus (RR 2.3, 1.2-4.7) (19). For the

Table 2. Pooled Relative Risk of Second Primary Malignancy in Thyroid Carcinoma Patients Treated with Radioactive Iodine Relative to Those Not Treated With RAdioactive Iodine

\begin{tabular}{lccc}
\hline Type of SPM & $\begin{array}{c}\text { Relative } \\
\text { risk }\end{array}$ & $\begin{array}{c}\text { 95\% Confidence } \\
\text { interval }\end{array}$ & p-value \\
\hline Any SPM & 1.19 & $1.04,1.36$ & 0.010 \\
Bladder & 1.19 & $0.51,2.78$ & 0.690 \\
Breast & 0.86 & $0.64,1.16$ & 0.324 \\
Central nervous system & 1.74 & $0.73,4.17$ & 0.213 \\
Colon and rectum & 1.16 & $0.77,1.75$ & 0.472 \\
Digestive tract & 1.17 & $0.88,1.54$ & 0.28 \\
Kidney and renal pelvis & 1.39 & $0.71,2.72$ & 0.338 \\
Leukemia & 2.50 & $1.13,5.53$ & 0.024 \\
Lung & 1.50 & $0.86,2.60$ & 0.151 \\
Melanoma (skin) & 0.86 & $0.43,1.70$ & 0.655 \\
Stomach & 1.66 & $0.74,3.72$ & 0.220 \\
\hline
\end{tabular}

The data pooled are from the SEER study by Brown et al. (18) and the European study by Rubino et al. (19). The total number of thyroid cancer survivors included in the pooled analysis is 16,502 .

SPM, second primary malignancy. 


\begin{tabular}{ccccccc} 
Study & \multicolumn{4}{c}{ Statistics for each study } & & RR and 95\% Cl \\
\cline { 2 - 6 } & RR & $\begin{array}{c}\text { Lower } \\
\text { limit }\end{array}$ & $\begin{array}{c}\text { Upper } \\
\text { limit }\end{array}$ & p-Value & \\
SEER 2008 & 1.18 & 0.95 & 1.47 & 0.143 & \\
Europe 2003 & 1.20 & 1.01 & 1.42 & 0.034 & \\
POOLED ESTIMATE & 1.19 & 1.04 & 1.36 & 0.010 &
\end{tabular}

FIG. 2. Fixed effects meta-analysis of the relative risk of any second primary malignancy in thyroid cancer survivors treated with radioactive iodine compared to those not treated with radioactive iodine. CI, confidence interval; SEER, Surveillance, Epidemiology, and End Results program of the National Cancer Institute.

outcome of salivary gland malignancies, a pooled analysis was not possible as there were no SPM events in the control group of the SEER study (18), and an RR could not be calculated. There was insufficient data available from one or both included studies for the calculation of pooled RR of other types of cancers. We were unable to calculate the pooled RR for multiple myeloma after RAI treatment, as the RR reported in the European study did not correspond with the reported 95\% CI (reported as RR 1.5, 95\% CI 0.3-0.7) and we did not receive a response to an e-mail request for clarification from the corresponding author. As for the SEER study (18), it is worthy of mention that for the outcome of stomach cancer, the standardized incidence rate (observed or expected) of stomach cancer was found to be increased in thyroid cancer survivors compared to the general population $(3.85,95 \% \mathrm{CI}$ 1.54-7.92); however, when we transformed the data in this study to an RR of stomach cancer in thyroid cancer survivors treated with radioisotopes compared to thyroid cancer survivors not treated with radioisotopes, the RR was 3.53 (95\% CI 0.80-21.13), which was not significantly elevated. The wider CIs in the RR calculation reflect the relative rarity of such tumors in thyroid cancer survivors, with or without a history of RAI treatment. On pooling data from the European (19) and SEER (18) studies, the risk of stomach cancer was not found to be significantly elevated in thyroid cancer survivors treated with RAI compared to those not treated with RAI. Similarly, for the outcome of mesothelioma in the SEER study (18), the standardized incidence rate for thyroid cancer survivors treated with radioisotopes compared to the general population was 3.10 (95\% CI, 1.34-6.11), whereas we calculated the RR to be $1.50(0.02-117.74)$ when compared to thyroid cancer survivors not treated with RAI. The RR of mesothelioma was not reported in the European study (19).

\section{Discussion}

In a prior review, we reported that thyroid cancer survivors have an increased risk of developing an SPM relative to the general population (17). In particular the risk of the following types of cancers was found to be elevated in thyroid cancer survivors relative to the general population: adrenal, bone and joints, breast, brain and central nervous system, colon and colorectal, kidney, leukemia, multiple myeloma, nonHodgkin's lymphoma, prostate, salivary gland, soft tissue sarcoma, and stomach (17). In the current review, we have learned that thyroid cancer survivors treated with RAI are at slightly increased risk of developing a second cancer compared to those not treated with RAI. In particular, the risk of leukemia is elevated in thyroid cancer survivors treated with RAI compared to those not treated with RAI. However, prior RAI exposure does not appear to explain the increased risk SPM of the breast, central nervous system, colon and rectum, kidney, and stomach, in thyroid cancer survivors compared to the general population (17). Furthermore, we did not observe a significantly increased risk of bladder or digestive system cancers in RAI-treated thyroid cancer survivors compared to those not treated with RAI. The most important limitations of our pooled analysis is the limited follow-up period of primary studies (as SPMs may occur decades after primary cancer treatments), and relatively large losses to follow-up in the European cohorts (approximately 20\%) (19). Moreover, we were unable to examine the relationship between cumulative RAI dose activity and the risk of SPMs in a formal metaregression analysis, since RAI dose activities were not reported in the SEER study (18). In the European study, Rubino et al. (19) reported that increasing cumulative activity of RAI administered was positively linearly associated with an increased risk of both solid tumors and leukemias.

It is important to highlight that the excess absolute risk of SPMs attributable to RAI treatment in thyroid cancer is likely to be small. For example, overall in the SEER study by Brown et al. (18) approximately $7 \%$ of thyroid cancer survivors developed a second cancer after a median follow-up period of 8.6 years. Also, in the European study by Rubino et al. (19), approximately $8 \%$ of thyroid cancer survivors were diagnosed with a second cancer after a mean follow-up of 13 years. Thus, assuming an approximate 5- to 10-year absolute risk of around 7-8\% for SPMs after a diagnosis of thyroid cancer and assuming a pooled RR of SPMs of 1.19 with RAI treatment, we estimate that the absolute risk of any SPM should be about $1 \%$ higher in individuals treated with RAI compared to those not treated with RAI. Furthermore, in the SEER study by Brown et al. (18), $0.23 \%(68 / 30,278)$ of thyroid cancer survivors developed leukemia after a median follow-up period of 8.6 years. Also, in the European study by Rubino et al. (19), $0.26 \%$ (18/6841) of thyroid cancer survivors were diagnosed with leukemia after a mean follow-up of 13 years. Thus, assuming an approximate 5- to 10-year absolute risk of around $0.23-$ $0.26 \%$ for leukemia after a diagnosis of thyroid cancer and assuming a pooled RR of leukemia of 2.50 with RAI treatment, we estimate that the absolute risk of leukemia should be about $0.4 \%$ higher in individuals treated with RAI compared to those not treated with RAI. An important limitation of these calculations is the limited follow-up period of the studies available for pooled analysis, and the fact that young thyroid cancer survivors may survive for decades after the diagnosis. This issue may be particularly relevant if certain tumors (such as solid tumors) have a longer latency period from time of initial treatment to development of SPM relative to hematologic malignancies. Thus, the risk of tumors latency period beyond about 5-10 years may be underestimated in our calculations.

The topic of RAI treatment for thyroid cancer and SPMs has been recently discussed in a narrative review by Ron (20). In this narrative review (20), some of the studies cited were the same as in our review $(18,19)$ but multiple other papers $(21-$ 26) were also tabulated (20). One of the main reasons why we included fewer studies in this review compared to the paper by Ron (20) was the restriction of inclusion of overlapping study populations from our study. Specifically, three of the 
studies cited in the recent narrative review by Ron were prior analyses from the SEER database (23-26) and were excluded from our meta-analysis as they overlapped with Brown et al. (18), which provided the most directly relevant information for our study question and the most recently updated data. Also, in the narrative review by Ron (20), a paper by Berthe et al. (21) on a French study population was cited, but this was excluded from our review as the region of study overlapped that of the larger, multi-center European study by Rubino et al. (19). A paper by Verkooijen et al. (22) from the Netherlands, was cited in the narrative review by Ron (20) but was excluded from our systematic review on methodologic grounds because it lacked of a control group of thyroid cancer patients not treated with RAI. The presence of strict inclusion criteria addressing a specific question and the avoidance of duplicate data are important features of systematic reviews.

There are multiple reports on the associations of oncologic medical and radiation therapies with increasing risk of subsequent SPMs (27-32). It is known that both chemotherapy and radiotherapy can induce leukemia, although the risks after administration of cytotoxic drugs are considered to be greater (27). It has been observed that following treatment with alkylating agent chemotherapy, the incidence of leukemia begins to increase at around 1 to 2 years and peaks at 5 to 10 years post-treatment; thereafter, it decreases (27). Platinum compounds and topoisomerase II inhibitors have also been reported to be associated with an increased risk of subsequent leukemia (27). The risk of leukemia following radiation treatment is thought to be about two orders smaller than that following chemotherapy (27). The incidence of leukemia following radiation peaks at approximately 5 to 9 years after exposure and subsequently declines (27). The incidence of secondary solid tumors after radiation treatment peaks much later than leukemias, with latency periods of 10 or more years after initial treatment (27). The risk of SPMs following radioisotope treatment has not been as clearly defined, hence the importance of reports cited in this review $(18,19)$. It is interesting to note that the reported RR of any SPM in breast cancer survivors treated with radiation (RR 1.2, 95\% CI 1.1-1.3) (32), is very similar to that observed in our pooled analysis for thyroid cancer survivors (RR 1.19, 95\% CI 1.04, 1.36). Furthermore, the RR of leukemia in breast cancer survivors treated with radiation (RR 1.8, 95\% CI 1.2-2.8) (32) is also not dissimilar to that observed in our pooled analysis for thyroid cancer survivors $(2.50,95 \%$ CI 1.13, 5.53). These findings suggest that SPM risk attributable to radioisotope treatment may be more similar to that of other forms of radiation therapy than to that of chemotherapeutic agents.

The strengths of this study include the use of rigorous systematic review methods and meta-analytic techniques to retrieve and pool relevant data from a relatively large number of individual patients ( $n=16,502$ in pooled analyses). Furthermore, our findings were robust using fixed and random effects models for pooling of data. Some limitations of this study include the following: the small number of studies included, the lack of a pooled quantitative examination of the relationship between dose activity of RAI and SPM risk (due to a lack of information on dose activities in the study by Brown et al. [18]), relatively short follow-up periods in the included studies, an English language restriction for inclusion, exclusion of unpublished data, the inherent methodologic limitations of the included studies (such as losses to follow-up, lack of data on thyroid cancer disease stage, selective reporting of outcomes, a lack of information on other important factors [such as family history, lifestyle practices, or any antecedent radiation exposure]), and statistical heterogeneity of pooled analyses. The statistical heterogeneity may be due to unstudied factors such as variability in administered dose activities, dose activities adsorbed, lifestyle factors, and individual genetic susceptibility to second cancers.

In conclusion, thyroid cancer survivors treated with RAI are at increased risk of developing SPMs relative to those not treated with RAI, albeit the absolute excess risk is estimated to be relatively small over the 5 to 10 years following diagnosis. Further research is needed to validate these findings with longer follow-up and in additional populations. It is also important to better define the relationships between dose activity administered, thyroid cancer disease stage, family history, and lifestyle factors with SPM risk in thyroid cancer survivors. Future research examining genetic susceptibility for SPM risk after RAI treatment is also needed.

\section{Acknowledgments}

The authors would like to thank Shoba Subramanian for assistance with retrieval and screening of some of the abstracts and papers in this review. The authors would also like to acknowledge the assistance of Rajeev Kashyap and Shahryar Murshed for in the data abstraction process. The authors would also like to thank Nofisat Ismaila for assistance with data entry. A. Sawka and this project are supported by the Canadian Institutes of Health Research New Investigator Program (CNI-80701). This study is also supported in part by the University Health Network Thyroid Research Centre Endowment Fund and the Connaught New Staff Matching Grant of the University of Toronto. This work was presented in abstract form at the 79th Annual Meeting of the American Thyroid Association in Chicago, Illinois, October 2, 2008.

\section{Disclosure Statement}

The authors have no commercial associations to declare that might create a conflict of interest in connection with this manuscript.

\section{References}

1. Canadian Cancer Society, National Cancer Institute of Canada, Statistics Canada, Public Health Agency of Canada 2008 Canadian Cancer Statistics 2008. Available at http:// www.cancer.ca/Canada-wide/About $\% 20$ cancer/Cancer $\%$ 20statistics/Canadian\%20Cancer\%20Statistics.aspx?sc_lang = en. Accessed November 5, 2008.

2. American Cancer Society 2008 Cancer Facts and Figures. Available at http://www.cancer.org/docroot/stt/stt_0.asp? from $=$ fast. Accessed November 5, 2008.

3. Davies L, Welch HG 2006 Increasing incidence of thyroid cancer in the United States. JAMA 295:2164-2167.

4. Hayat MJ, Howlader N, Reichman E, Edwards BK 2007 Cancer statistics, trends, and multiple primary cancers from the Surveillance, Epidemiology, and End Results (SEER) program. Oncologist 12:20-37.

5. Cancer Research UK 2008 Thyroid Cancer. Available at http://info.cancerresearchuk.org/cancerstats/types/thyroid/ incidence/. Accessed October 16, 2008. 
6. Verdecchia A, Francisci S, Brenner H, Gatta G, Micheli A, Mangone L, Kunkler I, for the Eurocare-4 Working Group 2007 Recent cancer survival in Europe: a 2000-02 period analysis of EUROCARE-4 data. Lancet Oncol 8:784-796.

7. Edwards BK, Howe HL, Ries LA, Thun MJ, Rosenberg HM, Feigal EG, Edwards BK 2002 Annual report to the nation on the status of cancer, 1973-1999, featuring implications of age and aging on U.S. cancer burden. Cancer 94:2766-2792.

8. Park SH, Suh EH, Chi JG 1988 A histopathologic study on 1,095 surgically resected thyroid specimens. Jpn J Clin Oncol 18:297-302.

9. Cooper DS, Doherty GM, Haugen BR, Kloos RT, Lee SL, Mandel SJ, Mazzaferri EL, McIver B, Sherman SI, Tuttle RM, for the American Thyroid Association Guidelines Task Force 2006 Management guidelines for patients with thyroid nodules and differentiated thyroid cancer. Thyroid 16:109-142.

10. National Cancer Institute 2006 Thyroid Cancer (PDQ): Treatment. Available at http://www.cancer.gov/cancertopics/ $\mathrm{pdq} /$ treatment/thyroid/healthprofessional/allpages. Accessed January 21, 2008.

11. Pacini F, Schlumberger $M$, Dralle H, Elisei R, Smit JW, Wiersinga W, for the European Thyroid Cancer Task Force 2006 European consensus for the management of patients with differentiated thyroid carcinoma of the follicular epithelium. Eur J Endocrinol 154:787-803.

12. American Association of Clinical Endocrinologists, American College of Endocrinology, American Association of Endocrine Surgeons 2007 AACE/AAES medical/surgical guidelines for clinical practice: management of thyroid carcinoma. Endocr Pract 7:202-220.

13. British Thyroid Association, Royal College of Physicians 2007 Guidelines for the management of thyroid cancer. 2nd ed. Available at http://www.british-thyroid-association.org. Accessed January 21, 2008.

14. National Comprehensive Cancer Network 2008 NCCN Clinical Practice Guidelines in Oncology: Thyroid Carcinoma. Available at http://www.nccn.org/professionals/ physician_gls/PDF/thyroid.pdf. Accessed Oct 20, 2008.

15. Pacini F, Castagna MG, Brilli L, Jost L, on behalf of the ESMO Guidelines Working Group 2008 Differentiated thyroid cancer: ESMO clinical recommendations for diagnosis, treatment and follow-up. Ann Oncol 19 (Suppl 2):ii99-ii101.

16. Jonklaas J, Sarlis NJ, Litofsky D, Ain KB, Bigos T, Brierley JD, Cooper DS, Haugen BR, Ladenson PW, Magner J, Robbins J, Ross DS, Skarulis M, Maxon HR, Sherman SI 2006 Outcomes of patients with differentiated thyroid carcinoma following initial therapy. Thyroid 16:1229-1242.

17. Subramanian S, Goldstein DP, Parlea L, Thabane L, Ezzat S, Ibrahim-Zada I, Straus S, Brierley JD, Tsang RW, Gafni A, Rotstein L, Sawka AM 2007 Second primary malignancy risk in thyroid cancer survivors: a systematic review and metaanalysis. Thyroid 17:1277-1288.

18. Brown AP, Chen J, Hitchcock YJ, Szabo A, Shrieve DC, Tward JD 2008 The risk of second primary malignancies up to three decades after the treatment of differentiated thyroid cancer. J Clin Endocrinol Metab 93:504-515.

19. Rubino C, de Vathaire F, Dottorini ME, Hall P, Schvartz C, Couette JE, Dondon MG, Abbas MT, Langlois C, Schlumberger M 2003 Second primary malignancies in thyroid cancer patients. Br J Cancer 89:1638-1644.

20. Ron E 2008 Treatment for thyroid cancer as a risk factor for a second malignancy. Available at http://thyroid.org/ professionals/publications/clinthy/clinthy_v202.pdf. Accessed November 24, 2008. Clinical Thyroidology 20(2):3-4.
21. Berthe E, Henry-Amar M, Michels JJ, Rame JP, Berthet P, Babin E, Icard P, Samama G, Galateau-Sallé F, Mahoudeau J, Bardet S 2004 Risk of second primary cancer following differentiated thyroid cancer. Eur J Nucl Med Mol Imaging 31:685-691.

22. Verkooijen RB, Smit JW, Romijn JA, Stokkel MP 2006 The incidence of second primary tumors in thyroid cancer patients is increased, but not related to treatment of thyroid cancer. Eur J Endocrinol 155:801-806.

23. Bhattacharyya N, Chien W 2006 Risk of second primary malignancy after radioactive iodine treatment for differentiated thyroid carcinoma. Ann Otol Rhinol Laryngol 115:607-610.

24. Chuang SC, Hashibe M, Yu GP, Le AD, Cao W, Hurwitz EL, Rao JY, Neugut AI, Zhang ZF 2006 Radiotherapy for primary thyroid cancer as a risk factor for second primary cancers. Cancer Lett 238:42-52.

25. Ronckers CM, McCarron P, Ron E 2005 Thyroid cancer and multiple primary tumors in the SEER cancer registries. Int J Cancer 117:281-288.

26. Sandeep TC, Strachan MW, Reynolds RM, Brewster DH, Scélo G, Pukkala E, Hemminki K, Anderson A, Tracey E, Friis S, McBride ML, Kee-Seng C, Pompe-Kirn V, Kliewer EV, Tonita JM, Jonasson JG, Martos C, Boffetta P, Brennan P 2006 Second primary cancers in thyroid cancer patients: a multinational record linkage study. J Clin Endocrinol Metab 91:1819-1825.

27. Travis LB 2006 The epidemiology of second primary cancers. Cancer Epidemiol Biomarkers Prev 15:2020-2026.

28. Howard R, Gilbert E, Lynch CF, Hall P, Storm H, Holowaty E, Pukkala E, Langmark F, Kaijser M, Andersson M, Joensuu H, Fossa SD, Allan JM, Travis LB 2008 Risk of leukemia among survivors of testicular cancer: a population-based study of 42,722 patients. Ann Epidemiol 18:416-421.

29. Neglia JP, Robison LL, Stovall M, Liu Y, Packer RJ, Hammond S, Yasui Y, Kasper CE, Mertens AC, Donaldson SS, Meadows AT, Inskip PD 2006 New primary neoplasms of the central nervous system in survivors of childhood cancer: a report from the Childhood Cancer Survivor Study. J Natl Cancer Inst 98:1528-1537.

30. Constine LS, Tarbell N, Hudson MM, Schwartz C, Fisher SG, Muhs AG, Basu SK, Kun LE, Ng A, Mauch P, Sandhu A, Culakova E, Lyman G, Mendenhall N 2008 Subsequent malignancies in children treated for Hodgkin's disease: associations with gender and radiation dose. Int J Radiat Oncol Biol Phys 72:24-33.

31. Hodgson DC, Gilbert ES, Dores GM, Schonfeld SJ, Lynch CF, Storm H, Hall P, Langmark F, Pukkala E, Andersson M, Kaijser M, Joensuu H, Fossa SD, Travis LB 2007 Long-term solid cancer risk among 5-year survivors of Hodgkin's lymphoma. J Clin Oncol 25:1-11.

32. Yu GP, Schantz SP, Neugut AI, Zhang ZF 2006 Incidences and trends of second cancers in female breast cancer patients: a fixed inception cohort-based analysis (United States). Cancer Causes Control 17:411-420.

Address reprint requests to: Anna M. Sawka, M.D., Ph.D., FRCPC

Toronto General Hospital 200 Elizabeth St.

Eaton North 12-212

Toronto, Ontario, M5G 2C4

Canada

E-mail: sawkaam@yahoo.com 
